inant. Consequently, the stable system found in the real world consists of a mosaic of microsites each of which may be in a different state of successional development or dominant maturation. The whole system may, nevertheless, approximate to the Odum ideal in terms of its energy and nutrient balance sheet.

This state of affairs is well documented in a recent study by Forcier (Science, 189, 808; 1975) of the canopy tree species in a forest in New Hampshire. Within this forest, $90 \%$ of the trees belonged to one of three species, namely beech (Fagus grandifolia), sugar maple (Acer saccharum) and yellow birch (Betula alleghaniensis). Forcier observed 400 randomly chosen plots within this forest over a period of three years and paid particular attention to the population dynamics of the seedlings of these species. Mortality of beech seedlings was found to be high initially but declined with age, thus average life-expectancy increased. Birch, on the other hand, had a fairly constant mortality whatever the seedling age. Sugar maple was intermediate in its population dynamics. Also, beech seedlings were found to vary little in their density whilst birch seedling densities varied considerably and average density was more than five times that of beech. These data suggest that birch is behaving as an opportunist in the composition of the forest.

Association analyses between the seedlings and the canopy trees showed that sugar-maple seedlings were associated with birch canopies and beech seedlings with sugar maple canopies. Evidently the three species are related in a cyclic successional series in which disturbance (or death of a mature tree) leads to birch invasion, sugar maple fares well beneath the birch canopy, eventually replacing it, and finally beech invades and replaces sugar maple. The roughly equal abundance of the three species of tree in the canopy is indicative of the importance of this cyclic microsuccession in what appears superficially to be a stable, 'climax' forest.

Forcier calls this a "near climax ecosystem', but this is rather misleading, since it suggests that the system falls short of the ideal. In a sense it does, for the community consists of a mosaic of sites, most of which are in different stages of the cyclic development. Nevertheless, the forest as a whole complies with many of the demands of the Odum ideal. There is no net increase in biomass, so gross ecosystem production is equalled by ecosystem respiration. Also the total input of nutrients from all sources (meteorological, geological and biological) must be equalled by total outputs. Reality fails to live up to the ideal only in that the 'stable' system contains species which cannot regenerate efficiently beneath their own canopy and which one therefore tends to associate with successional stages instead of the climax. Rather than call this forest a 'near climax', let us be ready to modify further the climax dogma and admit that climax is a carousel.

\section{What drives the solar cycle?}

\section{from Nigel Weiss}

IAU Symposium No. 71 on Basic Mechanisms of Solar Activity was held in Prague on August 25-29, 1975.

THE Sun is the only star that can be observed in detail and any theory of stellar structure must be tested against these observations. Unfortunately, conventional models cannot explain the low measured neutrino flux or the excitation of oscillations in the Sun: as I. W. Roxburgh (Queen Mary College, London) put it, "solar physics has forced the stellar physicist to think again." Over the past few years solar physics has developed rapidly. Observationally, it has become possible to resolve structures with scales smaller than the photosphere granulation, while theory is advancing from simple mechanisms to detailed computations.

The solar cycle is apparently typical of stars with convective envelopes and

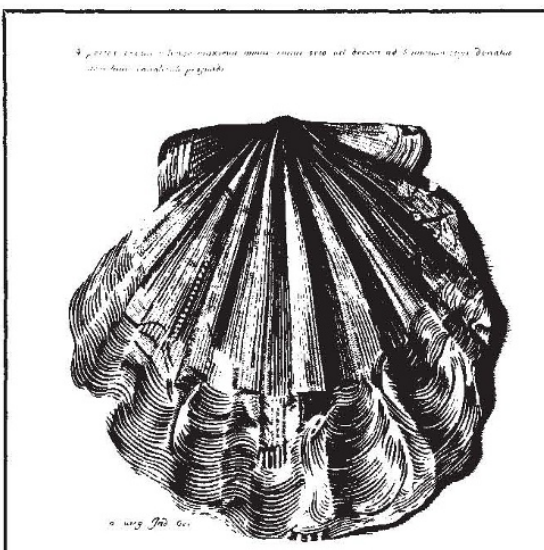

Reproduction of the illustration of the earliest figured and described fossil from America. From Lister's (1687) Historiae Conchyliorum Liber III. Lister's entire description is with each figure and not in a separate text. Say (1824) mentions this figure as being his species Pecten jeffersonius. (Reproduced from "Chesapecten, a new genus of Pectinidae (Mollusca: Bivalvia) from the Miocene and Pliocene of Eastern North America"-Geol. Surv. Prof. Pap. 861
O. C. Wilson (Hale Observatories) has found similar behaviour in several latetype stars. Magnetic fields on the Sun have been observed in profuse detail, from satellites and from the ground; indeed, we have far less information about fields in the Earth's core, which is shielded by the mantle. It is generally accepted that the cycle is maintained by an oscillatory dynamo yet $E$. N. Parker (University of Chicago) emphasised that many features of this process are still poorly understood.

The orthodox model is a dynamo driven by a combination of differential rotation and helical motion in the outer convective zone. A dipole-like field is wound round the Sun by differential rotation and the resulting toroidal field is twisted by helical convection to produce a reversed poloidal field. Samples, particularly of the strong toroidal field, pop through the surface in active regions. This process can be represented by mean field electrodynamics: F. Krause and K.-H. Rädler (Zentralinstitut für Astrophysik, Potsdam) described the procedure of separating large scale mean fields from small scale fluctuating fields and then averaging over space or time. Turbulent convection provides an enhanced eddy diffusivity and, through helicity (the $\alpha$ -effect), generates the poloidal field. Turbulence also produces fluctuating fields which, in the Sun, may be 100 times greater than the mean field itself. M. Stix (Göttingen University) discussed numerical solutions of the linear dynamo equations. With carefully chosen distributions of helicity and angular velocity, these yield remarkably accurate reproductions of the actual solar cycle.

Mean field electrodynamics is not perfect, however. The growth of the field is limited by non-linear effects that are not properly incorporated in the theory. Moreover, the procedure of averaging and smoothing cannot be justified for giant convective cells and highly concentrated fields. J. O. Stenflo (Lund University) has established that more than $90 \%$ of the photospheric magnetic flux is concentrated into small regions, a few hundred kilometres across, with fields of around 1.500 gauss. Outside these regions, except in pores and sunspots, only weak, irregular fields are detected. To describe such a jagged field distribution, a more elaborate theory is needed.

Unfortunately, the pattern of motion in the deep convective zone remains unclear. R. Howard and H. Yoshimura (Hale Observatories) have accumulated Doppler measurements of the differential rotation which also provide some evidence for the existence of giant cells extending through the whole depth of the convective zone. W. J. Wagner (Sacramento Peak Observatory) showed 